\title{
An Iris Recognition and Detection System Implementation
}

\author{
Olatunbosun A., Bamigboye 0
}

\begin{abstract}
There was big interest in the use of biometrical identification techniques, such as iris, face, fingerprints, ears, and significant technological developments and to enhance safety issues. The application varies depending on the location, based on the resources. They are used in safety at airports, border safety, criminal investigation, and so on. This study focuses on iris-based biometric technology. Biometric technology based on the iris diaphragm is the most reliable and acceptable among other biometric technologies. In this study, we developed the IRIS graphic user interface and attempted to use the streamlined segmentation technique to create a simpler and efficient way to detect iris. The 'Matlab ' software tool is being used to fix the recognized issues when implementing the produced code using suitable new algorithms. The proposed system is not just used to eliminates noises but also enables the border between the iris and the pupil to be correctly established. Results are saved in a computer with the corresponding model steps are performed using neural networks and synthesis algorithms. Pattern compatibility uses the appropriate metric to compare custom patterns with database patterns. The match option shows the measure of similarity between two diaphragm patterns. Finally, it is a strong level of trust that determines whether the user is authenticated or defined. As a binary template referred to as iris code the output of the Gabor wavelet (real and imaginary) is quantized as a stage. The FAR and FRR resulting from that are $0.001 \%$ and $37,880 \%$.
\end{abstract}

Keywords: Recognition, FRR and FAR, IRIS, Gabor and Detection.

\section{INTRODUCTION}

This iris is very precise, safe and functionally unique and stable over its whole lifetime compared to other biometric data [1]. The oldest and most reliable technique of airport identification and authentication, forensic services, computer safety and improved safety has been biometric systems. Also, the iris recognition systems remove the limitation of the facial recognition system and makes it more stable than other techniques. It is the most effective and precise biometric identifier. The Iris recognition program describes the human eye image and then divided and normalized [3]. The main technique for identifying systems is based on biometric information which is Iris Recognition. Some important physical features of a person including Keystroke pattern, gait, iris biometric, signature all have a strong, distinctive texture, new-born and longevity and are very stable because they are stable throughout a person's life and are widely recognized as a detection system [8-9].

Revised Manuscript Received on April 20, 2020.

*Correspondence Author

Olatunbosun A*., Department of Electrical and Electronic Engineering, University of Ibadan, Nigeria.

Bamigboye O, Department of Electrical and Electronic Engineering, Federal Polytechnic OFFA, Kwara State.

(c) The Authors. Published by Blue Eyes Intelligence Engineering and Sciences Publication (BEIESP). This is an open access article under the CC BY-NC-ND license (http://creativecommons.org/licenses/by-nc-nd/4.0/)
This is the best. Iris recognition includes iris segmentation, localizing, normalizing, coding, and comparison [2] In the past few years, according to [4] he acknowledged that the human perception based on characteristics of the iris has been recognized as highly unique, highly reliable, and un-invasive.

\section{REVIEW OF RELATED WORKS}

In recent years, the perception of the iris has been a popular research topic due its accuracy and almost complete recognition. There are three primary levels in the Iris recognition system: Pattern matching, Image pre-processing, feature extraction. To preserve a decent variety in the iris, the image must be semi-processed. Semi-processing of images is divided into three stages: localization of the iris, division of the iris and standardization of the iris. Innovative and extensive research was carried out exclusively when Daugman discovered the iris using the Gabor wavelet. Lately, distinct algorithms have been given a fresh route to other studies including [5-7]. Though, the complexities of time, memory and algorithm use in a broad variety of fields present some significant issues. This study contributes to the possible solution of many problems. In this study, feature extraction is performed using a continuous wave algorithm and a stochastic neural network algorithm.

\section{METHOD}

The structure suggested is depending on the system of recognition of the iris. Once the image is entered, the location and segmentation of the iris is done by means of Hough transformation. The transformation of the Gabor wavelet discovers the internal and outer edge of the iris, it then sees that the structure of the iris is a circle. Therefore, becomes a somewhat wider normalization of the recorded image of the iris. Using the Daugman Rubber SheetModel, the raw image of the iris is converted from Cartesian to polar. In the same standardized picture, the continuous wavelet transformation is used to achieve characteristics. The obtained attributes are for the category of neural networks only. This results are stored in a database. neural networks and fusion algorithms are used to implement the suitable template step. Pattern compatibility uses the appriopraite metric to compare custom patterns with database patterns. Therefore, a choice is produced with a high level of confidence to determine whether the person is legitimate or an imitator.

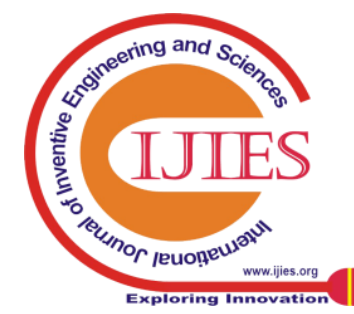




\section{An Iris Recognition and Detection System Implementation}

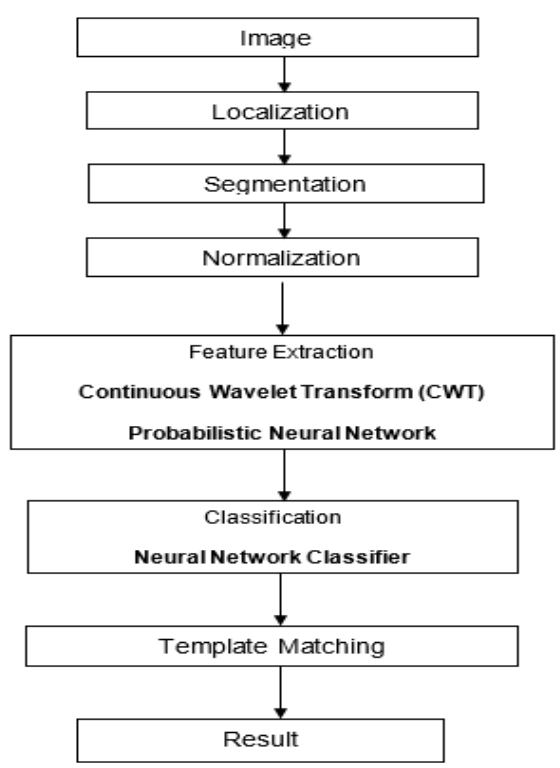

Figure. 1. The proposed iris recognition system framework

\section{RESULT AND DISCUSSION}

4.1 Result evaluation of Eye Boundary THE BOUNDARY SUCCESS RATE, REMOVING OF THE EYELASHES IS SHOWN IN TABLE1

Table1: Comparison of Iris Region

\begin{tabular}{|l|l|l|}
\hline Rate of success & Previous Method (\%) & Proposed Method (\%) \\
\hline Inner Boundary & 96.10 & 98.1 \\
\hline Outer Boundary & 90.51 & 92.9 \\
\hline Removal of eyelashes & 60.45 & 71.67 \\
\hline
\end{tabular}

The above table differentiate the achievement level in discovering, internal and external borders of the Iris by removing eyelashes from the proposed method using the method described earlier. By enhancing the image contrast and intensity, histogram equalization is regarded as a major method of improving picture.

AN HD COMPARISION OF EYE IMAGES OF THE SAME PERSON IN TABLE2

Table 2: HD Comparison of Iris Image

\begin{tabular}{|l|l|l|l|}
\hline Number & First Image & Second Image & HD \\
\hline 1 & 01 & 02 & 0.3854 \\
\hline 2 & 01 & 03 & 0.3007 \\
\hline 3 & 02 & 03 & 0.405 \\
\hline 4 & 01 & 04 & 0.4284 \\
\hline 5 & 02 & 04 & 0.4412 \\
\hline 6 & 02 & 05 & 0.44512 \\
\hline 7 & 02 & 06 & 0.41654 \\
\hline 8 & & 08 & 0.43124 \\
\hline & & & \\
\hline
\end{tabular}

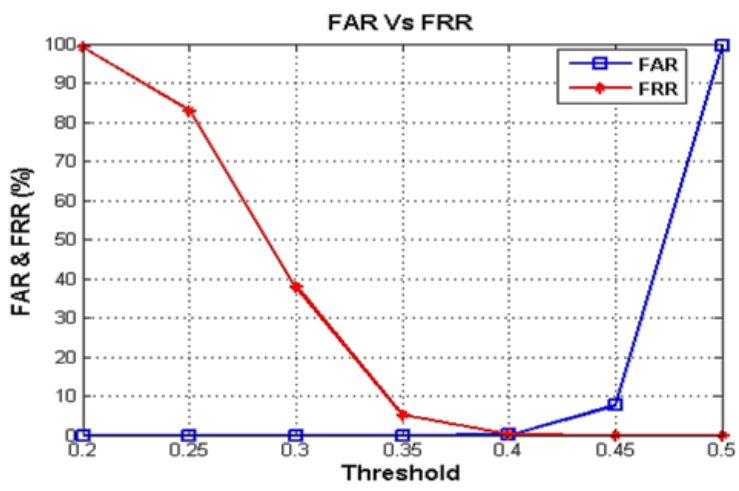

Figure 2: Comparison of FAR and FRR

FAR and FRR for another threshold value in Figure2 The above image demonstrates the proportion of FAR and FRR with the CASIA database's distinct threshold value. A reduced threshold value may reduce FAR and boost FRR.

Table 2. Iris Comparison of Recognition Score from Traditional Segment Model with and Without the Proposed Model of Detection

\begin{tabular}{|l|l|l|}
\hline Different Matchup & $\begin{array}{l}\text { Matching score of } \\
\text { Previous iris segment } \\
\text { Model }\end{array}$ & $\begin{array}{l}\text { Matching score of } \\
\text { Proposed Detection } \\
\text { Model }\end{array}$ \\
\hline M12 & 0.050 & 0.050 \\
\hline M13 & 0.064 & 0.061 \\
\hline M14 & 0.060 & 0.057 \\
\hline M15 & 0.075 & 0.053 \\
\hline M16 & 0.098 & 0.054 \\
\hline
\end{tabular}

Regardless of how to increase the percentage of unwanted iris eyelids, the outcome of the compared using the suggested technique is stable between 0.050 and 0.060 as demonstrated in Table 2. One case, that is. M16, less than half the evaluation, which corresponds to the technique suggested, compared with the current one, is provided by the suggested detection model. The researched findings shows that the detection model suggested, necessary for obtaining the characteristics of the iris, provides feature high accuracy of recognition.

\section{CONCLUSION AND RECOMMENDATION}

In the iris detection scheme, removal of eyelid is always an important stage. In the segmented iris region, the elliptical iris causes unwanted eyelids. This can be seen as an unwanted data that is noisy when extracting the functions of the iris. They have no important texture information. For iris, normalized via the eyelashes and the eyelids, the unique characteristics should be minimized. The algorithms presented in this study can minimize important pieces of unwanted information. Not only does the suggested system eliminate the existence of noisy components, it also enables you to determine the border between the iris and the pupil correctly. The stage quantization (real and imaginary) of the Gabor wavelet results represents the result as an Iris Code binary model. The resulting FAR and FRR are $0.001 \%$ and $37.880 \%$ respectively.

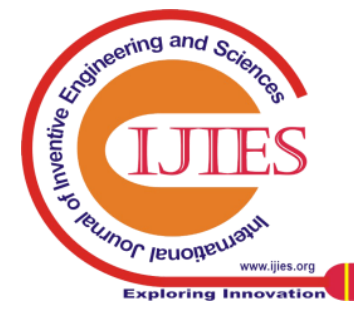




\section{REFERENCE}

1. Dolly. C, Shamik., T \& Ajay., K.(2012). A Survey: Feature Extraction Methods for Iris Recognition. International Journal of Electronics Communication and Computer Technology (IJECCT) Vol. 2, Issue 6. pp. 45-55.

2. Hugo, A. \& Heitor, S. (2013). A Genetic Programming Approach for Image Segmentation. Journal of Computational Intelligence in Image Processing. Retrieved from https://link.springer.com/chapter/10.1007\%2F978-3-642-306211_4page-1

3. Kandla., A.(2012). The use of Face Recognition Concept. International Journal of Soft Computing and Engineering. "ISSN: 2231-2307, Volume2, Issue-5, pp45-61

4. Khaw, P. (2012). Iris Recognition Technology: The Iris Technology for Improved Authentication. SANS Institute Infosec Reading Room.

5. OBAJE. S. (2010). Automatic Fingerprint and Toe print Recognition for Personal Identification and Forensic Application. Ph.D. thesis, University Of Ilorin. Nigeria

6. Roger, A. (2012). A process of Recognition of Human Iris: Fast Segmentation of the Iris Department of Computer Sciences, Florida Institute of Technology

7. Jaydeep., C.(2015). Face Recognition under Varying Illumination. International Journal of Research in advance Engineering, Vol.1 Issue-2, pp. 35-40

8. Jonathan., G, Aledir., S, Norian., M, Alex., F, \& Joao., M.(2011) Method for iris recognition based on its internal region. Institute of Mechanical Engineering and Industrial Management (INEGI), 4200465.

9. Vibha, S. \& Ramesh, N. (2015). Periocular and Iris Feature Encoding: A Survey. International Journal of Innovative Research in Computer and Communication Engineering. Vol. 3, Issue 1, pp 368374: Retrieved from 10.15680/ijircce.2015.0301023

\section{AUTHOR PROFILE}

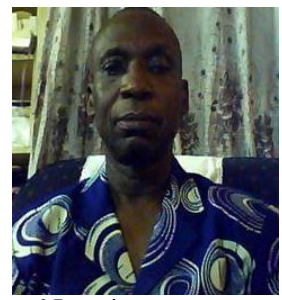

PROFESSOR Adeboye Olatunbosun, B.Sc. (Hons) (Teesside), MSc, Ph.D. (Manchester) MInstM\&C, MIEEE, MISA, MTWAMP, CEng Area of Specialization

Instrumentation and Process Automation, Industrial Reliability Analysis and Asset Management, industrial Process Optimization, and Integrated Renewal Energy analysis and design Multi-Component Flow Measurement

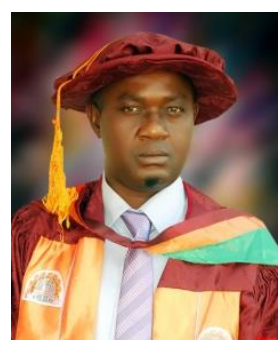

Bamigboye Oladayo Oladele, holds a B.Eng. degree in Electrical and Electronic Engineering from university of Ilorin Kwara State, Nigeria and Master's degree in the same field with specialization in instrumentation and control Engineering. He is presently rounding up his Ph.D. program in University of Ibadan, Nigeria. He has published many local and international journals and textbooks in his field. He is Chief Lecturer and presently the Head of Department of Electrical and Electronic Engineering in the Federal Polytechnic. Offa, Kwara State, Nigeria. He is married with children

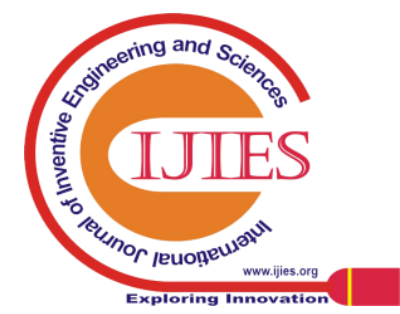

